\title{
Robust estimation of parallel robot dynamic parameters with interval analysis
}

\author{
Ph. Poignet ${ }^{1}$, N. Ramdani ${ }^{2}$, O. A. Vivas ${ }^{1}$ \\ ${ }^{1}$ Laboratoire d'Informatique, de Robotique et de Microélectronique de Montpellier (LIRMM), UMR CNRS 5506, \\ University Montpellier 2, 161 Rue Ada, 34392 Montpellier cedex 5, France. \\ $<$ vivas, poignet>@lirmm.fr \\ ${ }^{2}$ Centre d'Etude et de Recherche en Thermique, Environnement et Systèmes, \\ Université Paris XII- Val de Marne, ave G. de Gaulle, 94000 Créteil. \\ ramdani@univ-paris12.fr
}

\begin{abstract}
This paper deals with the application of interval analysis for outer bounding the physical parameters of parallel robots. The robot is modeled with classical Lagrange equation which leads to an inverse dynamic model linear with respect to the parameters. Assuming the error additive on input (motor torque), the problem is termed as a constraint propagation one and the solution is performed with a preconditionned Gauss-Seidel contractor modified in order to be used with over-determined linear systems. Experimental results are exhibited for a fully parallel robot with 4 degrees of freedom.
\end{abstract}

Keywords: outer enclosure, Gauss-Seidel contractor, parameters estimation, robots dynamics.

\section{INTRODUCTION}

Robust estimation of physical parameters (e.g. for robot dynamic model) are often necessary for computing robust model based control algorithms. This robust identification can be performed through bounded errors techniques (Walter and Pronzato, 1997) (Maksarov and Norton, 2002) (Poignet et al., 2003). Bounded errors may encompass significant structural errors that cannot be accounted for by random variables or noise errors without specifying any statistical properties. Among the bounded error methods, interval arithmetic provides efficient tools to compute guaranteed estimation or robust control (Jaulin et al., 2001) (Jaulin et al., 2002). In such a context, the solution is a set of parameter vector consistent with measurement data, prior error bounds and modeling hypotheses.

In this paper, the problem of dynamic robot parameter estimation is expressed with a model which is linear with respect to the physical parameters. The estimation problem is then stated as a linear interval constraint satisfaction problem (CSP). Interval fixed-point contractors make it then possible to compute a smallest box outer-bounding the solution set (Jaulin et al., 2001).

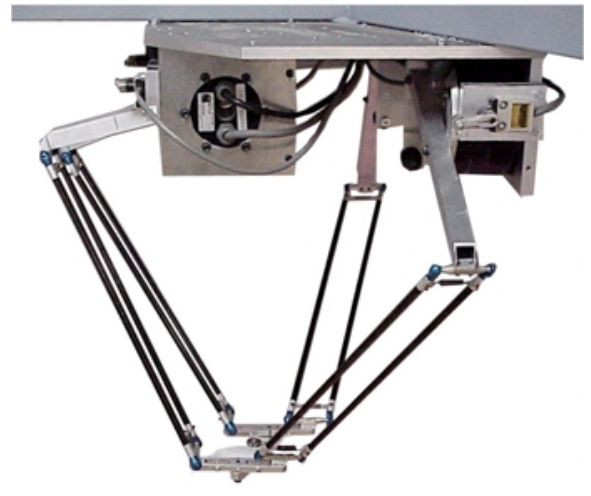

Fig. 1. H4 robot.

Experimental results are exhibited for a 4-dof parallel mechanism - the H4 robot - (Company and Pierrot, 1999; Pierrot et al, 2001). Figure 1 shows a photograph of the $\mathrm{H} 4$ parallel robot. This machine is based on 4 independent active chains between the base and the nacelle; each chain is actuated by a brushless direct drive motor fixed on the base and equipped with an incremental position encoder. Thanks to its design, the mechanism is able to provide high performance. However in order to achieve high speed and acceleration for pick-and-place applications or precise motion in machining tasks, advanced model based robust controllers are often required to increase the performances of the robot, which justify these works on guaranteed estimation.

The paper is organized as follows : Section 2 is dedicated to the geometric, kinematics and dynamic modelling of the H4 robot. Section 3 details the interval methods. Section 4 exhibits major experimental results on a fully parallel robot. Finally, conclusions are given in section 5 .

\section{MODELLING}

\subsection{Geometric and kinematics modelling}

The Jacobian matrix and the forward geometric model are required to compute the dynamic model (see section 2.2) 
(Khalil, and Dombre, 2002). Therefore we briefly present the way of computing the different relationship necessary to obtain these model and matrix. The design parameters of the robot are described on Figure 2 where the following parameters have been chosen:

$\alpha_{1}=0 ; \alpha_{2}=\pi ; \alpha_{3}=3 \pi / 2 ; \alpha_{4}=3 \pi / 2$

$\boldsymbol{u}_{1}=\boldsymbol{u}_{y} ; \boldsymbol{u}_{2}=-\boldsymbol{u}_{y} ; \boldsymbol{u}_{3}=\boldsymbol{u}_{x} ; \boldsymbol{u}_{4}=\boldsymbol{u}_{x}$

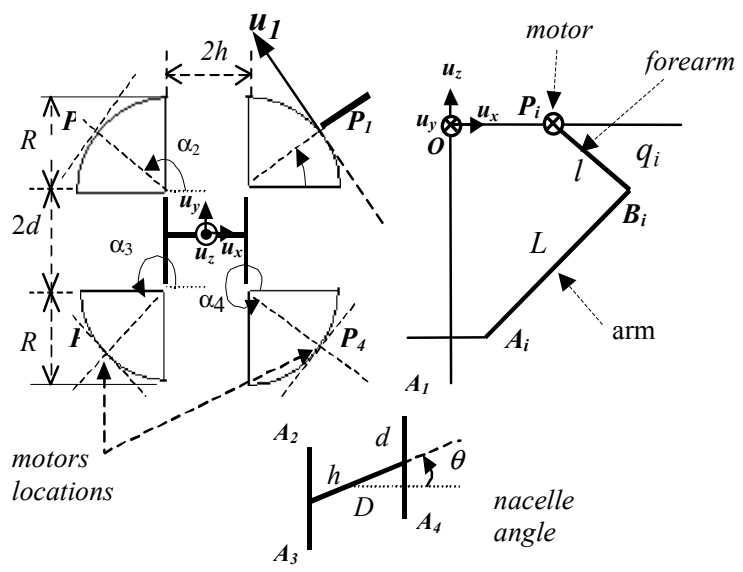

Fig. 2. Design parameters.

The angles $\alpha_{i}$ describe the position of the four motors, $L$ is the length of arms, $l$ is the length of the forearms, $\theta$ the nacelle's angle, and $d$ and $h$ are the half lengths of the " $\mathrm{H}$ forming the nacelle. $\boldsymbol{O}$ is the origin of the base frame and $D$ is the origin of the nacelle frame. $R$ gives the motor's position. The $\boldsymbol{A}_{\boldsymbol{i}} \boldsymbol{B}_{\boldsymbol{i}}$ segments represent the arms of the robot and $\boldsymbol{P}_{\boldsymbol{i}} \boldsymbol{B}_{\boldsymbol{i}}$ the forearm segments. The joint positions are represented by $q_{i}$.

To obtain the geometric model, we need to express the different points of the mechanical system with respect to the origin $\boldsymbol{O}$. The origin is fixed in the middle of the nacelle with the coordinates $(x, y, z)$. In the Cartesian space, the end effector position is given by $(x, y, z, \theta)$.

$$
\mathbf{O D}=\left[\begin{array}{lll}
x & y & z
\end{array}\right]^{T}
$$

The vector that joins the absolute origin $O$ and all of the forearms to the nacelle is:

$$
\boldsymbol{O} \boldsymbol{A}_{\boldsymbol{i}}=\boldsymbol{O D}+\boldsymbol{D} \boldsymbol{A}_{\boldsymbol{i}}=\left[\begin{array}{l}
x \\
y \\
z
\end{array}\right]+\boldsymbol{D} \boldsymbol{A}_{\boldsymbol{i}}
$$

The $\boldsymbol{D} \boldsymbol{A}_{\boldsymbol{i}}$ segments can be expressed as:

$$
\begin{aligned}
\boldsymbol{D A}_{1} & =\left[\begin{array}{c}
h \cos \theta \\
h \sin \theta+d \\
0
\end{array}\right] ; \boldsymbol{D A}_{2}=\left[\begin{array}{c}
-h \cos \theta \\
-h \sin \theta+d \\
0
\end{array}\right] \\
\boldsymbol{D A}_{3} & =\left[\begin{array}{c}
-h \cos \theta \\
-h \sin \theta-d \\
0
\end{array}\right] ; \boldsymbol{D A}_{4}=\left[\begin{array}{c}
h \cos \theta \\
h \sin \theta-d \\
0
\end{array}\right]
\end{aligned}
$$

Moreover, the vector that links the absolute origin and all of the arms to the forearms is:

$$
O B_{i}=O P_{i}+P_{i} B_{i}
$$

with:

$$
\boldsymbol{P}_{\boldsymbol{i}} \boldsymbol{B}_{\boldsymbol{i}}=\left[\begin{array}{c}
l \cos q_{i} \cos \alpha_{i} \\
l \cos q_{i} \sin \alpha_{i} \\
-l \sin q_{i}
\end{array}\right]
$$

and actuator locations are:

$$
\begin{gathered}
\boldsymbol{O P}_{1}=\left[\begin{array}{c}
h+R \cos \alpha_{1} \\
d+R \sin \alpha_{1} \\
0
\end{array}\right] ; \boldsymbol{O P}{ }_{2}=\left[\begin{array}{c}
-h+R \cos \alpha_{2} \\
d+R \sin \alpha_{2} \\
0
\end{array}\right] \\
\boldsymbol{O P}_{3}=\left[\begin{array}{c}
-h+R \cos \alpha_{3} \\
-d+R \sin \alpha_{3} \\
0
\end{array}\right] ; \boldsymbol{O P} \boldsymbol{P}_{4}=\left[\begin{array}{c}
h+R \cos \alpha_{4} \\
-d+R \sin \alpha_{4} \\
0
\end{array}\right]
\end{gathered}
$$

Finally, arms coordinates are given by:

$$
\mathbf{A}_{\mathbf{i}} \mathbf{B}_{\mathbf{i}}=\mathbf{A}_{\mathbf{i}} \mathbf{O}+\mathbf{O B} \mathbf{B}_{\mathbf{i}}
$$

The analytical forward position relationship is difficult to compute. Up to now, the simplest model we have got is a $8^{\text {th }}$ degree polynomial equation. The forward model is then computed iteratively using the classical formula:

$$
\mathbf{x}_{\mathrm{n}+1}=\mathbf{x}_{\mathrm{n}}+\mathbf{J}\left(\mathbf{x}_{\mathrm{n}}, \mathbf{q}_{\mathrm{n}}\right)\left[\mathbf{q}-\mathbf{q}_{\mathrm{n}}\right]
$$

Where $\mathbf{q}$ is the convergence point and $\mathbf{J}$ is the robot Jacobian matrix. If the mechanism is not in a singular configuration, this expression is derived as follows (Company and Pierrot, 1999; Pierrot et al, 2001):

$$
\mathbf{J}=\mathbf{J}_{\mathrm{x}}^{-1} \mathbf{J}_{\mathrm{q}}
$$

Where: 


$$
\begin{aligned}
& \mathbf{J}_{x}=\left[\begin{array}{llll}
A_{1} B_{1 x} & A_{1} B_{1 y} & A_{1} B_{1 z} & \left(\mathbf{D C} \mathbf{C}_{1} \mathbf{X} \mathbf{A}_{1} \mathbf{B}_{1}\right)_{z} \\
A_{2} B_{2 x} & A_{2} B_{2 y} & A_{2} B_{2 z} & \left(\mathbf{D C} \mathbf{C}_{1} \mathbf{X} \mathbf{A}_{2} \mathbf{B}_{2}\right)_{z} \\
A_{3} B_{3 x} & A_{3} B_{3 y} & A_{3} B_{3 z} & \left(\mathbf{D C} \mathbf{C}_{2} \mathbf{X} \mathbf{A}_{3} \mathbf{B}_{3}\right)_{z} \\
A_{4} B_{4 x} & A_{4} B_{4 y} & A_{4} B_{4 z} & \left(\mathbf{D C} \mathbf{C}_{2} \mathbf{X} \mathbf{A}_{4} \mathbf{B}_{4}\right)_{z}
\end{array}\right] \\
& \mathbf{J}_{q}=\operatorname{diag}\left(\left(\mathbf{P}_{i} \mathbf{B}_{i} \times \mathbf{A}_{i} \mathbf{B}_{i}\right) \cdot \mathbf{u}_{\mathbf{m i}}\right) \quad, \quad i=1, \ldots 4
\end{aligned}
$$

$\boldsymbol{D} \boldsymbol{C}_{\boldsymbol{i}}(i=\{1,2\})$ is the distance between the center of the nacelle and the center of the half lengths of the " $\mathrm{H}$ " that forms the nacelle.

\subsection{Dynamic modelling}

In first approximation, the dynamic model is computed by considering physical dynamics. Indeed, the drive torques are mainly used to move the motor inertia, the fore-arms and the arms and the nacelle equipped with a machining tool. Because of the design, the fore-arm inertia can be considered as a part of the motor inertia and the arm (manufacturing in carbon materials) effects are neglected [8], [9]. A simple friction model is added considering viscous and Coulomb friction.

If $\Gamma_{\text {mot }}$ is the $(4 \mathrm{x} 1)$ actuator torque vector, the basic equation of dynamics can be written as :

$$
\Gamma_{m o t}=\mathbf{I}_{m o t} \ddot{\mathbf{q}}+\mathbf{J}^{T} \mathbf{M}(\ddot{\mathbf{x}}-G)+\mathbf{F}_{v} \dot{\mathbf{q}}+\mathbf{F}_{s} \operatorname{sign}(\dot{\mathbf{q}})
$$

where $\mathbf{I}_{\mathbf{m o t}}$ represents the motor's inertia matrix including the forearm's inertia, $\mathbf{M}$ a matrix containing the mass of the nacelle and its inertia, $\dot{\mathbf{q}}$ is the $(4 \times 1)$ joint velocity vector, $\ddot{\mathbf{q}}$ is the $(4 \times 1)$ joint acceleration vector, $\ddot{\mathbf{x}}$ is the (4x1) vector of cartesian accelerations $\left[\begin{array}{llll}\ddot{x} & \ddot{y} & \ddot{z} & \ddot{\theta}\end{array}\right]^{T}$, and $G$ the gravity constant. Thanks to the design, the forearm's inertia is taken into account in the motor's inertia. $\mathbf{F}_{\mathbf{v}}$ are the viscous friction coefficients and $\mathbf{F}_{\mathbf{c}}$ are the Coulomb friction.

With:

$$
\begin{aligned}
\mathbf{I}_{\text {mot }} & =\left[\begin{array}{cccc}
I_{\text {mot } 1} & 0 & 0 & 0 \\
0 & I_{\text {mot } 2} & 0 & 0 \\
0 & 0 & I_{\text {mot } 3} & 0 \\
0 & 0 & 0 & I_{m o t}
\end{array}\right] \\
\mathbf{M}_{\text {mot }} & =\left[\begin{array}{cccc}
M_{\text {nac }} & 0 & 0 & 0 \\
0 & M_{\text {nac }} & 0 & 0 \\
0 & 0 & M_{\text {nac }} & 0 \\
0 & 0 & 0 & I_{b c}
\end{array}\right]
\end{aligned}
$$

The dynamic equation can be rewritten as a relation linear in the dynamic parameters. By introducing

$$
\begin{aligned}
& \mathbf{J}^{\mathrm{T}}=\left[\begin{array}{ll}
\mathbf{J}_{43} & \mathbf{J}_{4}
\end{array}\right] \text {, it follows: }
\end{aligned}
$$

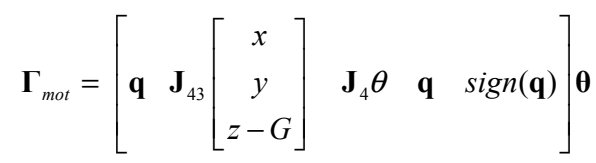

where $\boldsymbol{\theta}$ is the vector of parameters:

$$
\begin{aligned}
& \boldsymbol{\theta}=\left[\begin{array}{lllllllll}
I_{m o t 1} & I_{m o t 2} & I_{m o t 3} & I_{m o t 4} & M_{n a c} & I_{b c} \\
F_{v 1} & F_{v 2} & F_{v 3} & F_{v 4} & F_{s 1} & F_{s 2} & F_{s 3} & F_{s 4}
\end{array}\right]^{T}
\end{aligned}
$$

Only the torque input $\boldsymbol{\Gamma}_{\text {mot }}$ and motor position $\mathbf{q}$ are directly measured. As acceleration measurement $\ddot{\mathbf{x}}$ is not available, $\ddot{\mathbf{x}}$ is evaluated by:

$$
\ddot{\mathbf{x}}=\mathbf{J} \ddot{\mathbf{q}}+\dot{\mathbf{J}} \dot{\mathbf{q}}
$$

where $\mathbf{J}$ depends on $\mathbf{x}$ and $\mathbf{q}$, is computed using a central difference algorithm.

\section{PARAMETER BOUNDING}

\subsection{Bounded error context}

When the statistical properties of the random variable used to model the actual disturbances acting on model inputs or outputs remain unattainable, it is still possible to compute values for the bounds between the output of a model $y_{k}^{m}(\cdot)$ and some actual measurements $y_{k}$. Indeed, the sensors used for data measurements are frequently characterized with a prior maximum measurement error. Under the hypothesis of additive noise, actual model output can be related to actual data as follows:

$$
y_{k}=y_{k}^{m}\left(\boldsymbol{\theta}^{*}\right)+\varepsilon_{k}^{*}, k=1 \ldots N
$$

where $N$ is the number of observations and $\boldsymbol{\theta}^{*}$ is the unknown true parameter vector to be identified and $\left\{\varepsilon^{*}\right\}$ an output error sequence assumed to be stationary and bounded but otherwise unknown. The error sequence thus satisfies the following inequality:

$$
\forall k=1 \ldots N,-1 \leq \varepsilon_{k}^{*} \leq 1
$$

This description is known as the standard form with a normalized error, and it is always possible to transform the case where the upper and lower prior error bounds are different to such a form.

A $(p \times 1)$ parameter vector $\boldsymbol{\theta}$ is said to be feasible, if and only if the output error is enclosed in the prior bounds. 
Consequently, the issue of the bounded-error set estimation is to compute the set, known as the posterior feasible set, defined as

$$
S=\left\{\boldsymbol{\theta} \in Q \mid \forall k=1 \ldots N,-1 \leq y_{k}-y_{k}^{m}(\boldsymbol{\theta}) \leq 1\right\}
$$

where the prior parameter search space $Q \subseteq \mathbb{R}^{p}$.

When the model is linear in parameter, which is the case of the robot inverse dynamic model, it is written as:

$$
y_{k}^{m}=\mathbf{d}_{k}^{T} \boldsymbol{\theta}
$$

The parameters set compatible with the datum at observation $k$ is a strip $\Pi_{k}$ defined by:

$$
\Pi_{k}=\left\{\boldsymbol{\theta} \in \mathbb{R}^{p}|| \mathbf{y}_{k}-\mathbf{d}_{k}^{T} \boldsymbol{\theta} \mid \leq 1\right\}
$$

The posterior feasible set is the intersection of a prior search space $Q$ and $N$ strips $\Pi_{k}$ :

$$
S=\left\{\boldsymbol{\theta} \in Q|\forall k=1 \ldots N,| y_{k}-\mathbf{d}_{k}^{T} \boldsymbol{\theta} \mid \leq 1\right\}
$$

Consequently, characterizing the solution parameter vectors set (25) consists on solving the following Constraint Satisfaction Problem (CSP)

$$
H:(\mathbf{W} \boldsymbol{\theta}-\mathbf{Y}=0, \quad \mathbf{Y} \in[\mathbf{Y}], \quad \boldsymbol{\theta} \in Q)
$$

where $\quad \mathbf{W}=\left[\mathbf{d}_{1}^{T}, \mathbf{d}_{2}^{T}, \ldots, \mathbf{d}_{N}^{T}\right]^{T}, \quad[\mathbf{Y}]=[\mathbf{Y}-\mathbf{1}, \mathbf{Y}+\mathbf{1}] \quad$ and $\mathbf{Y}=\left[\mathbf{y}_{1}, \mathbf{y}_{2}, \ldots, \mathbf{y}_{\mathbf{N}}\right]^{T}$. One can write equivalently

$$
H:(\mathbf{W} \boldsymbol{\theta}-[\mathbf{Y}]=0, \quad \boldsymbol{\theta} \in Q)
$$

The solution vector $[\boldsymbol{\theta}]$ is given as an interval vector. The width of each component of the interval solution vector indicates the uncertainty associated with the identified physical parameter.

In fact, due to the large number of data available, the system described by (27) is over-determined. It can however be re-written with square matrices by using the following method introduced in (Rump, 2002):

$$
\mathrm{H}:(\mathbf{A X}-[\mathbf{b}]=0, \quad \mathbf{X} \in X)
$$

where

$$
\mathbf{A}=\left[\begin{array}{cc}
\mathbf{W} & -\operatorname{eye}(N) \\
\operatorname{zeros}(p) & \mathbf{W}^{T}
\end{array}\right]
$$

$$
[\mathbf{b}]=\left[\begin{array}{c}
{[\mathbf{Y}]} \\
\operatorname{zeros}(p, 1)
\end{array}\right]
$$

where eye $(N)$ is an $(N \times N)$ identity matrix, $\operatorname{zeros}(p)$ is an $(p \times p)$ matrix of zeros and $z \operatorname{eros}(p, 1)$ an $(p \times 1)$ vector of zeros. The solution vector is then given by the first $p$ components of the $[\mathbf{X}]$ vector.

\subsection{Fixed-point contractors}

Interval CSPs can be solved with contractors. An operator $C_{H}$ is a contractor for the CSP $H$ if it satisfies:

$$
\begin{array}{lll}
\forall[\mathbf{x}] \in Q & C_{H}([\mathbf{x}]) \subset[\mathbf{x}] & \text { (contractance) } \\
\forall[\mathbf{x}] \in Q & C_{H}([\mathbf{x}]) \cap S=[\mathbf{x}] \cap S & \text { (correctness) }
\end{array}
$$

where $\cap$ is the intersection of two boxes (Jaulin et al., 2001).

A solver for a $\operatorname{CSP} \mathrm{H}:(\mathbf{f}(\mathbf{x})=\mathbf{0}, \mathbf{x} \in[\mathbf{x}])$ is an algorithm $\Psi$ such that:

$$
\mathbf{f}(\mathbf{x})=\mathbf{0} \Leftrightarrow \mathbf{x}=\Psi(\mathbf{x})
$$

According to the fixed point theorem and using (32), if the series $\mathbf{x}_{k+1}=\Psi\left(\mathbf{x}_{k}\right)$ converges towards $\mathbf{x}_{\infty}$, then $\mathbf{x}_{\infty}$ shall contain the solution of $H$.

For linear square systems, a well known method is given by the following interval Gauss-Seidel contractor, where the matrices $[\mathbf{A}]$ and $[\mathbf{b}]$ can be interval ones.

Algorithm $C_{G S}$ :

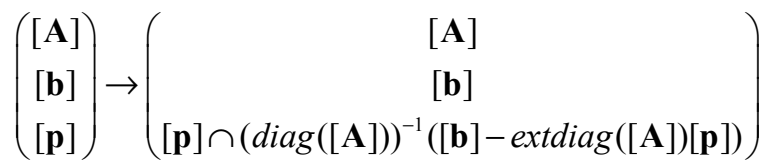

Where: $\mathbf{A}=\operatorname{diag}(\mathbf{A})+\operatorname{ext} \operatorname{diag}(\mathbf{A})$.

Remark: All the diagonal element of the A matrix must be non-null.

In practice, a more efficient version of the Gauss-Seidel contractor can be obtained by preconditioning the $\mathbf{A}$ matrix. The new algorithm is presented in the following:

and 


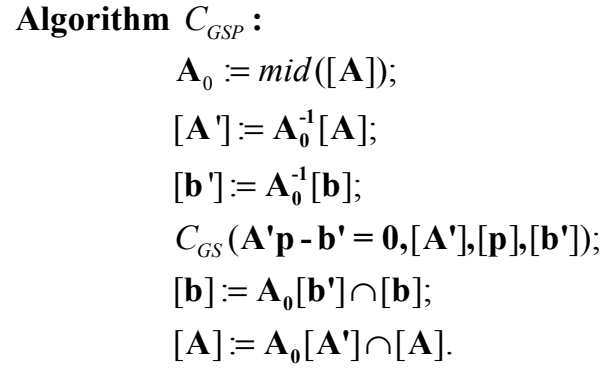

Where mid stands for the point value taken at the centre of the boxes and $C_{G S}$ is the Gauss-Seidel contractor (Jaulin et al., 2001).

\section{EXPERIMENTAL RESULTS}

\subsection{Experimental data}

Joint position $\mathbf{q}$ and the current reference $\mathbf{V}_{\mathbf{T}}$ (the control input) are collected at a $1000 \mathrm{~Hz}$ sample rate while the robot is tracking exciting trajectories containing both slow (for friction) and high dynamics (for inertia). These trajectories ensure a low condition number. The identification is performed by using a closed-loop joint PI control. The torques are computed using a linear relationship between torque $\Gamma_{\text {mot }}$ and voltage $\mathbf{V}_{\mathbf{T}}$ where $\mathrm{G}_{\mathbf{T}}$ is the amplifier gain:

$$
\Gamma_{\text {mot }}=G_{T} V_{T}
$$

Joint velocities and accelerations for computing the regressor are estimated by a band pass filtering of the position. The band pass filtering is obtained by the product of a low pass filter in both the forward and the reverse direction (Butterworth) and a derivative filter obtained by a central difference algorithm, without phase shift. A parallel filtering is implemented to reject the high frequency ripples of the measured motor torques. Practical aspects of the derivative estimation and data filtering are completely detailed in (Poignet and Gautier, 2001).

\subsection{Estimated parameters}

The prior bounds on motor torques are tuned by taking into account prior information on motors. They were chosen prior to the computation as $10 \%$ of measurement range of the torques $( \pm 15 \mathrm{Nm})$.

Table 1 contains the estimated parameter boxes. Prior values for motor inertia and nacelle mass and inertia are known by design. The initial boxes are equal to $[0.0,2.0]$. Table 2 exhibits a comparison with the weighted least square estimation results (Vivas et al., 2003). The estimated parameter boxes contain the prior values as well as the weighted least square solution. The estimated bounds are tight enough to be considered as a good estimation.
Table 1. Estimated parameter boxes. Values are expressed in USI.

\begin{tabular}{ccc}
\hline Parameter & Estimated boxes & $\begin{array}{c}\text { A priori } \\
\text { values }\end{array}$ \\
\hline $\mathrm{I}_{\mathrm{mot} 1}$ & {$\left[\begin{array}{lll}0.0009, & 0.0238\end{array}\right]$} & 0.012 \\
$\mathrm{I}_{\mathrm{mot} 2}$ & {$\left[\begin{array}{lll}0.0044, & 0.0257\end{array}\right]$} & 0.012 \\
$\mathrm{I}_{\mathrm{mot} 3}$ & {$\left[\begin{array}{lll}0.0096, & 0.0320\end{array}\right]$} & 0.012 \\
$\mathrm{I}_{\mathrm{mot} 4}$ & {$\left[\begin{array}{lll}0.0098, & 0.0319\end{array}\right]$} & 0.012 \\
$\mathrm{M}_{\mathrm{nac}}$ & {$\left[\begin{array}{lll}0.7650, & 1.2338\end{array}\right]$} & 1.0 \\
$\mathrm{I}_{\mathrm{bc}}$ & {$\left[\begin{array}{lll}0.0000, & 0.0071\end{array}\right]$} & 0.0008 \\
$\mathrm{~F}_{\mathrm{v} 1}$ & {$\left[\begin{array}{ll}0.0000, & 0.4690\end{array}\right]$} & $/$ \\
$\mathrm{F}_{\mathrm{v} 2}$ & {$\left[\begin{array}{ll}0.1733, & 0.2554\end{array}\right]$} & $/$ \\
$\mathrm{F}_{\mathrm{v} 3}$ & {$\left[\begin{array}{lll}0.0000, & 0.2856\end{array}\right]$} & $/$ \\
$\mathrm{F}_{\mathrm{v} 4}$ & {$\left[\begin{array}{ll}0.0000, & 0.2664\end{array}\right]$} & $/$ \\
$\mathrm{F}_{\mathrm{c} 1}$ & {$\left[\begin{array}{ll}0.0000, & 1.6068\end{array}\right]$} & $/$ \\
$\mathrm{F}_{\mathrm{c} 2}$ & {$\left[\begin{array}{ll}0.0000, & 1.6300\end{array}\right]$} & $/$ \\
$\mathrm{F}_{\mathrm{c} 3}$ & {$\left[\begin{array}{ll}0.2245, & 1.0952\end{array}\right]$} & $/$ \\
$\mathrm{F}_{\mathrm{c} 4}$ & {$\left[\begin{array}{ll}0.2264, & 1.1014\end{array}\right]$} & $/$ \\
\hline
\end{tabular}

Table 2. Weighted least square estimated parameters Values are expressed in USI.

\begin{tabular}{crr}
\hline Parameters & $\begin{array}{c}\text { Estimated } \\
\text { values }\end{array}$ & \multicolumn{1}{c}{$\% \sigma_{\hat{x} r}$} \\
\hline$I_{\text {motl }}$ & 0.0141 & 2.6286 \\
$I_{\text {mot } 2}$ & 0.0120 & 3.0444 \\
$I_{\text {mot } 3}$ & 0.0153 & 1.6939 \\
$I_{\text {mot } 4}$ & 0.0213 & 1.1933 \\
$M_{\text {nac }}$ & 1.0492 & 0.4236 \\
$I_{b c}$ & 0.0030 & 3.5049 \\
$F_{v 1}$ & 0.1636 & 5.6781 \\
$F_{v 2}$ & 0.0560 & 15.5674 \\
$F_{v 3}$ & 0.0930 & 6.5734 \\
$F_{v 4}$ & 0.0917 & 6.4301 \\
$F_{c 1}$ & 1.1453 & 2.0450 \\
$F_{c 2}$ & 1.0950 & 2.0563 \\
$F_{c 3}$ & 0.7222 & 2.8366 \\
$F_{c 4}$ & 0.9932 & 2.0451 \\
\hline
\end{tabular}

Where $\% \sigma_{\hat{x} r}$ stands for the relative standard deviation. Complete details of the computation are given in (Vivas et al., 2003). 


\section{CONCLUSION}

This paper exhibits relevant results for robust dynamic identification of parallel robots stated as an interval constraint satisfaction problem. The solution set is outerbounded with the pre-conditioned interval Gauss-Seidel contractor.

For the parallel robot under study, the estimated parameter boxes contain the prior values. The uncertainties derived for the parameter remain large.

Further work will concern $i$ ) the study of the influence of the prior error bounds on the posterior uncertainty, $i$ ) the possibility of accounting for data outliers and iii) the case where the regressor is no longer exact but subject to bounded uncertainty.

\section{REFERENCES}

Company O. and F. Pierrot (1999). A new 3T-1R parallel robot. ICAR'99, Tokyo, Japan, October 25-27, pp. 557562, 1999.

Jaulin L., Braems I. and Walter E. (2002), Interval methods for nonlinear identification and robust control, Proceedings of the IEEE 2002 Conference on Decision and Control, December 10 - 13, Las Vegas, USA.

Jaulin L., Kieffer M., Didrit O. and Walter E. (2001), Applied Interval Analysis, Springer-Verlag, London.

Khalil W. and E. Dombre (2002). Modeling, Identification and Control of Robots. Hermes Penton Science, London.

Lawson, C. and Hanson, C. (1974) Solving Least Squares Problems, Prentice-Hall.

Maksarov, D.G. and Norton, J.P. (2002), Computationally efficient algorithms for state estimation with ellipsoidal approximation, Int. J. Adapt. Control Signal Process., vol. 16, n6, pp. 411-434.

Pierrot F., F. Marquet, O. Company and T. Gil (2001). H4 Parallel Robot,: Modeling, Design and Preliminary Experiments. Proceedings of the 2001 IEEE International Conference one Robotics \& Automation, Seoul, Korea, May 21-26, 2001.

Poignet $\mathrm{Ph}$. and M. Gautier (2001). Extended kalman filtering and weighted least squares dynamic identification of robots. Control Engineering Practice, vol. 9/12, pp. 1361-1372.

Poignet Ph., Ramdani N. and Vivas A.O. (2003). Ellipsoidal estimation of parallel robot dynamic parameters, Proc. of the IEEE/RSJ Int. Conf. On Intelligent Robots and Systems (IROS), LasVegas, USA, October 27-31, 2003.

Rump S.M. (2002), INTLAB - Interval Laboratory, a Matlab toolbox for verified computations, V3.1, http://www.ti3.tu-harburg.de/rump/intlab/index.html.

Vivas O. A., Poignet Ph., Marquet F., Pierrot F. and Gautier M. (2003). Experimental dynamic identification of a fully parallel robot. To be published in Proceedings of the IEEE 2003 International Conference on Robotics and Automation, Taipei, Taïwan, September 14-19.

Walter, E. and Pronzato, L. (1997). Identification of parametric models from experimental data, Springer, London. 